05

\title{
Магнитокалорический эффект и эффект памяти формы в сплаве Гейслера $\mathrm{Mn}_{2} \mathrm{NiGa}$
}

\author{
(С) А.П. Каманцев ${ }^{1,2}$, Ю.С. Кошкидько ${ }^{1,3}$, Э.О. Быков ${ }^{4,5}$, В.С. Калашников ${ }^{1}$, А.В. Кошелев ${ }^{6,7}$, \\ А.В. Маширов ${ }^{1}$, И.И. Мусабиров ${ }^{8}$, М.А. Пауков ${ }^{2,9}$, В.В. Соколовский ${ }^{10}$ \\ ${ }^{1}$ Институт радиотехники и электроники им. В.А. Котельникова РАН, \\ Москва, Россия \\ 2 Лаборатория новых магнитных материалов Балтийского федерального университета им. И. Канта, \\ Калининград, Россия \\ ${ }^{3}$ Institute of Low Temperature and Structure Research of PAS, \\ 50-422 Wrocław, Poland \\ ${ }^{4}$ Hochfeld-Magnetlabor Dresden (HLD-EMFL) Helmholtz-Zentrum Dresden-Rossendorf, \\ 01328 Dresden, Germany \\ ${ }^{5}$ Institut für Festkörper- und Materialphysik, Technische Universität Dresden, \\ 01062 Dresden, Germany \\ ${ }^{6}$ Московский государственный университет им. М.В. Ломоносова, \\ Москва, Россия \\ ${ }^{7}$ Институт экспериментальной минералогии РАН, \\ Черноголовка, Россия \\ ${ }^{8}$ Институт проблем сверхпластичности металлов РАН, \\ Уфа, Россия \\ ${ }^{9}$ Faculty of Mathematics and Physics, Charles University, \\ 12116 Prague, Czech Republic \\ 10 Челябинский государственный университет, \\ Челябинск, Россия \\ E-mail: kaman4@gmail.com
}

Поступила в Редакцию 30 декабря 2019 г.

В окончательной редакции 30 декабря 2019 г.

Принята к публикации 10 января 2020 г.

\begin{abstract}
Представлены исследования функциональных свойств сплава Гейслера $\mathrm{Mn}_{2} \mathrm{NiGa}$, выплавленного методом аргонно-дуговой плавки. Прямыми экспериментальными методами исследованы: электросопротивление, намагниченность, магнитокалорический эффект и эффект памяти формы в широком диапазоне температур $100-400 \mathrm{~K}$. Обнаружен обратный магнитокалорический эффект как в мартенситной, так и в аустенитной фазах, не испытывающий насыщения в импульсном магнитном поле до 50 Т. Получены значения обратимой деформации в сплаве до 0.35\% при изгибающих механических нагрузках до $247 \mathrm{MPa}$.
\end{abstract}

Ключевые слова: магнитокалорический эффект, эффект памяти формы, сплавы Гейслера, импульсные магнитные поля

DOI: $10.21883 /$ FTT.2020.05.49236.12M

\section{1. Введение}

В последние годы сплавы Гейслера системы $\mathrm{Mn}_{2} \mathrm{NiZ}$ $(\mathrm{Z}=\mathrm{Ga}, \mathrm{In}, \mathrm{Sn}, \mathrm{Sb})$ привлекли большой интерес исследователей, как ферромагнитные сплавы с эффектом памяти формы (ЭПФ) и полуметаллы (ферро- или ферримагнетики), вследствие интересных физических свойств и потенциального применения для создания магнитных актюаторов и спинтронных устройств [1-10]. Исследования данных сплавов были простимулированы открытием магнитоиндуцируемых деформаций (4\%) в монокристалле $\mathrm{Mn}_{2} \mathrm{NiGa}$, обладающим термоупругим мартенситным фазовым переходом (ФП) вблизи комнатной температуры - $270 \mathrm{~K}$, и высокой температурой магнитного ФП - $588 \mathrm{~K}[1]$. Более того, сплав $\mathrm{Mn}_{2} \mathrm{NiGa}$ является ферримагнетиком в аустенитной (кубической) фазе в отличие от широко известного сплава $\mathrm{Ni}_{2} \mathrm{MnGa}$ [2]. Ферримагнитное упорядочение возникает вследствие антипараллельной ориентации магнитных моментов атомов $\mathrm{Mn}$, расположенных в различных подрешетках. Следует отметить, что классические соединения Гейслера $\mathrm{X}_{2} \mathrm{YZ}$ кристаллизуются в структуру типа $\mathrm{Cu}_{2} \mathrm{MnAl}$ с пространственной группой симметрии Fm3m (№ 225). Атомы X располагаются в позициях Вайкоффа 8c (1/4,1/4,1/4), атомы $\mathrm{Y}$ в позициях 4b $(1 / 2,1 / 2,1 / 2)$ и атомы $\mathrm{Z}$ в позициях $4 \mathrm{a}(0,0,0)$, что соответствует L2 1 структуpe, или иначе, данную структуру называют регулярной Гейслеровской структурой (РГС). На сегодняшний день известно более 1000 сплавов, которые кристаллизуются в РГС [3]. Помимо этого, сообщалось о примерно 100 сплавах со структурой близкой к регулярной это, так называемая, обратная Гейслеровская структура 
(ОГС), в которой половина атомов $\mathrm{X}$ обменивается своими позициями с атомами $\mathrm{Y}$ (пример, $\mathrm{Hg}_{2} \mathrm{CuTi}$ с пространственной группой симметрии $\mathrm{F} 43 \mathrm{~m}$ (№ 216)). Здесь, атомы $\mathrm{X}$ занимают позиции $4 \mathrm{~d}(1 / 4,1 / 4,1 / 4)$ и $4 \mathrm{~b}$ $(1 / 2,1 / 2,1 / 2)$, атомы $\mathrm{Y}-4 \mathrm{c}(3 / 4,3 / 4,3 / 4)$ и атомы $\mathrm{Z}-$ 4a $(0,0,0)$. Таким образом, различие между сплавами $\mathrm{Mn}_{2} \mathrm{NiZ}$ (с ОГС) и $\mathrm{Ni}_{2} \mathrm{MnZ}$ (с РГС) заключается в том, что в ОГС атомы Мn занимают две кристаллографически неэквивалентные позиции, в отличие от двух атомов $\mathrm{Ni}$ в обычной структуре. Неэквивалентность позиций атомов Mn приводит к более сложным магнитным структурам (например, к неколлинеарным магнитным состояниям).

Последующие экспериментальные исследования сплавов системы $\mathrm{Mn}_{2} \mathrm{NiGa}$, привели к обнаружению эффекта типа „спиновый замок“ в магнитосопротивлении для моно- и поликристаллических образцов вследствие антисайт беспорядка, при котором $13 \%$ позиций $\mathrm{Ga}$ занято атомами Mn [4]. Наличие обратного МКЭ в сплавах $\mathrm{Mn}_{2} \mathrm{NiGa}$ и $\mathrm{Mn}_{1.75} \mathrm{Ni}_{1.25} \mathrm{Ga}$ было косвенно продемонстрировано в работе [5]. Авторы показали, что изменение энтропии вблизи мартенситного ФП больше в сплаве $\mathrm{Mn}_{1.75} \mathrm{Ni}_{1.25} \mathrm{Ga}$, и МКЭ линейно возрастает с магнитным полем в обоих образцах. Результаты нейтронной дифракции показали, что обратный МКЭ вызван антиферромагнитным взаимодействием между атомами $\mathrm{Mn}$, занимающими неэквивалентные позиции [5]. Схожие экспериментальные и теоретические исследования сплавов семейства $\mathrm{Mn}_{2} \mathrm{NiZ}(\mathrm{Z}=\mathrm{Ga}, \mathrm{In}, \mathrm{Sn}, \mathrm{Sb})$ были выполнены в работах [1-10], так что анализируя современное состояние научных исследований сплавов Гейслера системы $\mathrm{Mn}_{2} \mathrm{NiZ}$, можно сказать, что данная область исследований является относительно новой и перспективной, что подтверждается наличием малого числа публикаций (начиная с 2005 г.), по сравнению с хорошо изученной системой сплавов Гейслера $\mathrm{Ni}_{2} \mathrm{MnZ}$.

\section{2. Объекты и методы исследования}

Методом аргонно-дуговой плавки был выплавлен слиток сплава Гейслера $\mathrm{Mn}_{2} \mathrm{NiGa}$ из элементов $\mathrm{Mn}, \mathrm{Ni}$ и $\mathrm{Ga}$ высокой чистоты. С целью получения однородного состава была выполнена семикратная переплавка слитка, полученного при выплавке. Анализ элементного состава, проведенный с помощью энерго-дисперсионного спектрометра X-Act Oxford Instruments, показал, что полученный слиток имеет состав $\mathrm{Mn}_{50.3} \mathrm{Ni}_{25.0} \mathrm{Ga}_{24.7}$ (ат.\%). В пределах точности рентгеноспектрального метода исследований, ликвации химического состава не обнаружено. Для анализа микроструктуры из слитка сплава был вырезан образец в форме пластины, и после полировки на абразивной бумаге с понижением зернистости, полировки на алмазной пасте образец был подвергнут электрополировке в растворе электролита $10 \% \mathrm{HCl}+90 \% \mathrm{C}_{4} \mathrm{H}_{9} \mathrm{OH}$. Анализ микроструктуры сплава выполнен на растровом электронном микроскопе в режиме регистрации обратно-отраженных электронов (BSE mode) с ускоряющим напряжением $20 \mathrm{kV}$.

Исследование ФП, лежащих выше 373 K выполнено с помощью дифференциальной сканирующей калориметрии (DSC) при нагреве образца со скоростью $5 \mathrm{~K} / \mathrm{min}$ до температуры $1273 \mathrm{~K}$. Термомагнитные исследования и электросопротивление образцов сплава проводились на установке Quantum Design PPMS-9T в диапазоне температур от 10 до $390 \mathrm{~K}$. Исследования ЭПФ вблизи комнатных температур проводились при помощи оригинальной методики, подробно описанной в [11], при механических нагрузках от 10 до $247 \mathrm{MPa.} \mathrm{Исследования}$ МКЭ проводились в Дрезденской лаборатории сильных магнитных полей (Hochfeld-Magnetlabor Dresden (HLDEMFL)) по методике, описанной в работе [12]. Для измерения температуры при этом использовалась дифференциальная термопара, свитая из проводов меди и константана (толщина одного провода без изоляции $-25 \mu \mathrm{m}$ ).

Попутно экспериментальным исследованиям свойств сплава $\mathrm{Mn}_{2} \mathrm{NiGa}$, в работе также были выполнены теоретические исследования основного состояния сплава $\mathrm{Mn}_{2} \mathrm{NiGa}$ в рамках теории функционала плотности. Расчеты электронной структуры выполнены с помощью метода проекционных присоединенных плоских волн (PAW), реализованного в программном пакете VASP $[13,14]$. Обменно-корреляционные эффекты рассмотрены в приближении обобщенного градиента (GGA) и параметризации Пердью-Бурке-Эрнзенхофа (PBE) [15]. В качестве расчетной ячейки выбраны 16-ти атомные кубические суперячейки РГС (Fm3m, № 225) и ОГС (F43m, № 216). В случае РГС рассмотрено ферромагнитное упорядочение (магнитные моменты атомов $\mathrm{Mn}$ и $\mathrm{Ni}$ сонаправлены), тогда как для ОГС ферромагнитное и ферримагнитное упорядочение (магнитные моменты атомов Mn, расположенных в различных подрешетках, ориентированы антипараллельно). Интегрирование по зоне Брюллиэна выполнено на сетке $8 \times 8 \times 8 k$-точек, автоматически сгененированной по схеме Монхроста-Пака. Энергия обрезания плоских волн и порог сходимости по энергии выбраны $800 \mathrm{eV}$ и $10^{-7} \mathrm{eV}$ соответственно.

\section{3. Экспериментальные результаты и их анализ}

Как известно, метод аргонно-дуговой плавки подразумевает выплавку сплавов на водоохлаждаемом медном тигле. При кристаллизации идет интенсивный рост кристаллов в слитке снизу-вверх. В связи с этим, для исследования микроструктуры был вырезан образец в форме пластины, плоскость которой параллельна росту кристаллов. Методом сканирующей электронной микроскопии в режиме регистрации обратно-отраженных электронов был проведен анализ целостности и качества микроструктуры сплава после выплавки. Микроструктура сплава в литом состоянии представлена на рис. 1. 
На изображении наблюдается отчетливый контраст, свидетельствующий о наличии вытянутых кристаллов шириной несколько сотен микрометров. При этом длина кристаллов может достигать нескольких миллиметров. На изображении также присутствуют области размером порядка $100 \mu \mathrm{m}$ с мелким контрастом, соответствующие областям мартенсита, что говорит о том, что мартенситное ФП в сплаве Гейслера $\mathrm{Mn}_{2} \mathrm{NiGa}$ реализуется вблизи комнатных температур.

Методом DSC проведен анализ ФП при температурах от 373 до $1273 \mathrm{~K}$ при нагреве. Соответствующие результаты представлены на рис. 2. На кривой наблюдаются два эндотермических пика, самый крупный

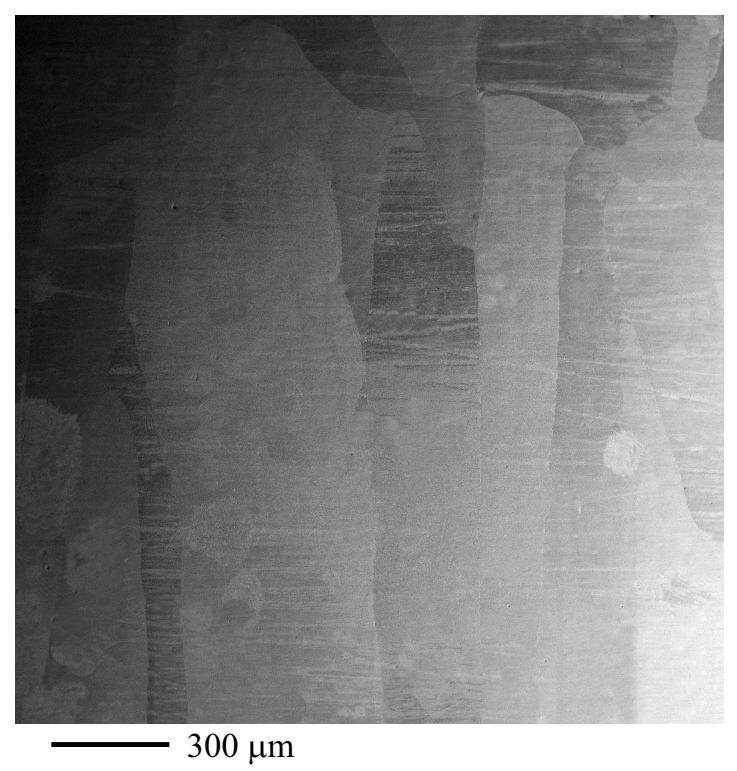

Рис. 1. Изображение микроструктуры сплава Гейслера $\mathrm{Mn}_{2} \mathrm{NiGa}$ в литом состоянии в режиме регистрации обратноотраженных электронов при комнатной температуре.

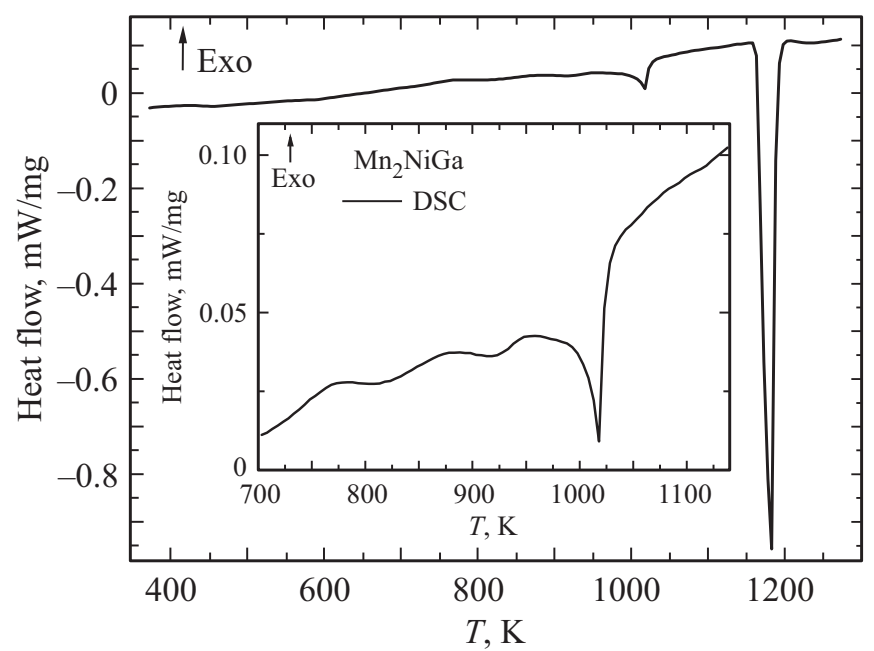

Рис. 2. Кривые DSC для литого образца сплава $\mathrm{Mn}_{2} \mathrm{NiGa}$, полученные при нагреве образца в интервале температур от 373 K до 1273 К. На вставке: выделенный участок в интервале $700-1140 \mathrm{~K}$.

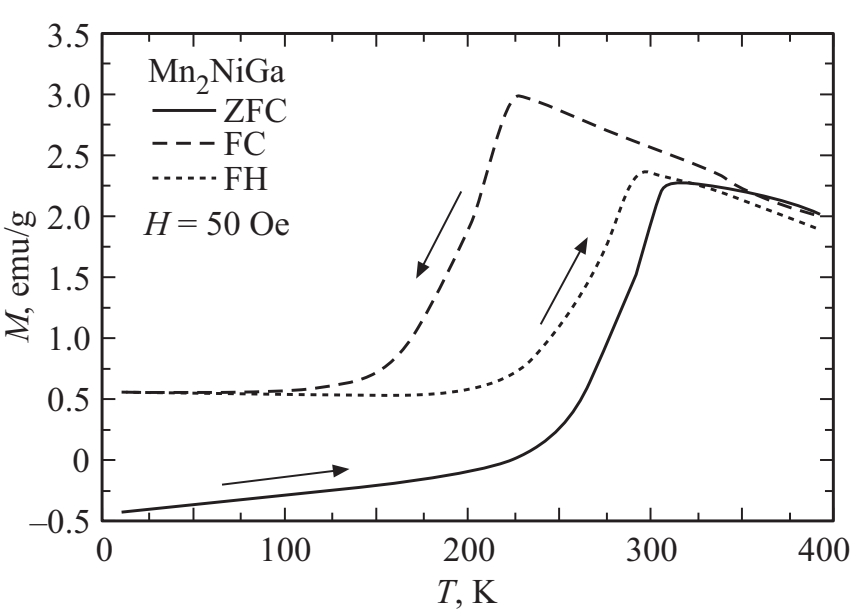

Рис. 3. Зависимость намагниченности образца сплава Гейслеpa $\mathrm{Mn}_{2} \mathrm{NiGa}$ в литом состоянии от температуры в магнитном поле 50 Ое по протоколу измерений ZFC-FC-FH.

пик соответствует точке плавления сплава при $1183 \mathrm{~K}$. На рис. 2 во врезке представлен участок кривой ДСК в интервале температур 700-1140 К, при этом пик при $1018 \mathrm{~K}$ соответствует ФП „Порядок-беспорядок“ из L2 1 в В2 фазу.

Зависимость намагниченности образца сплава $\mathrm{Mn}_{2} \mathrm{NiGa}$ (в литом состоянии) от температуры в слабом магнитном поле 50 Ое, полученная по протоколу $\mathrm{ZFC}-\mathrm{FC}-\mathrm{FH}$, представлена на рис. 3. Наличие отрицательной магнитной восприимчивости в слабом поле в мартенситной фазе при нагреве после охлаждении без поля обусловлено ферримагнитным упорядочением как мартенситной, так и аустенитной фаз.

С целью подтверждения ферримагнитного упорядочения в аустенитной и мартенситной фазе, дополнительно были выполнены первопринципные расчеты электронной структуры $\mathrm{Mn}_{2} \mathrm{NiGa}$ в аустенитной и мартенситной фазе. На рис. 4, $a$ приведена разница энергии кристаллических структур 225 и 216 группы симметрии для $\mathrm{Mn}_{2} \mathrm{NiGa}$ по отношению к выгодной структуре в зависимости от изменения параметра решетки и учета различного магнитного упорядочения. Можно видеть, что ОГС с ферримагнитным упорядочением в равновесном состоянии является энергетически выгодной по сравнению с регулярной структурой. Равновесный параметр кубической решетки ОГС с ферримагнитным упорядочением составляет $5.85 \mathrm{~A}$. Зависимости полной энергии от тетрагонального искажения с/а для $\mathrm{Mn}_{2} \mathrm{NiGa}$ с учетом различных магнитных конфигураций, посчитанные для суперячеек РГС и ОГС, изображены на рис. 4, $b$. В данном случае, приведены изменения энергий по отношению к равновесной энергии ОГС с ферримагнитным упорядочением. На рис. $4, b$ виден минимум энергии при соотношении $\mathrm{c} / \mathrm{a} \approx 1.3$ для случая ОГС, что говорит о возможном мартенситном ФП из кубического аустенита в тетрагональный мартенсит в ферримагнитном состоянии. Теоретические значения полного магнитного 

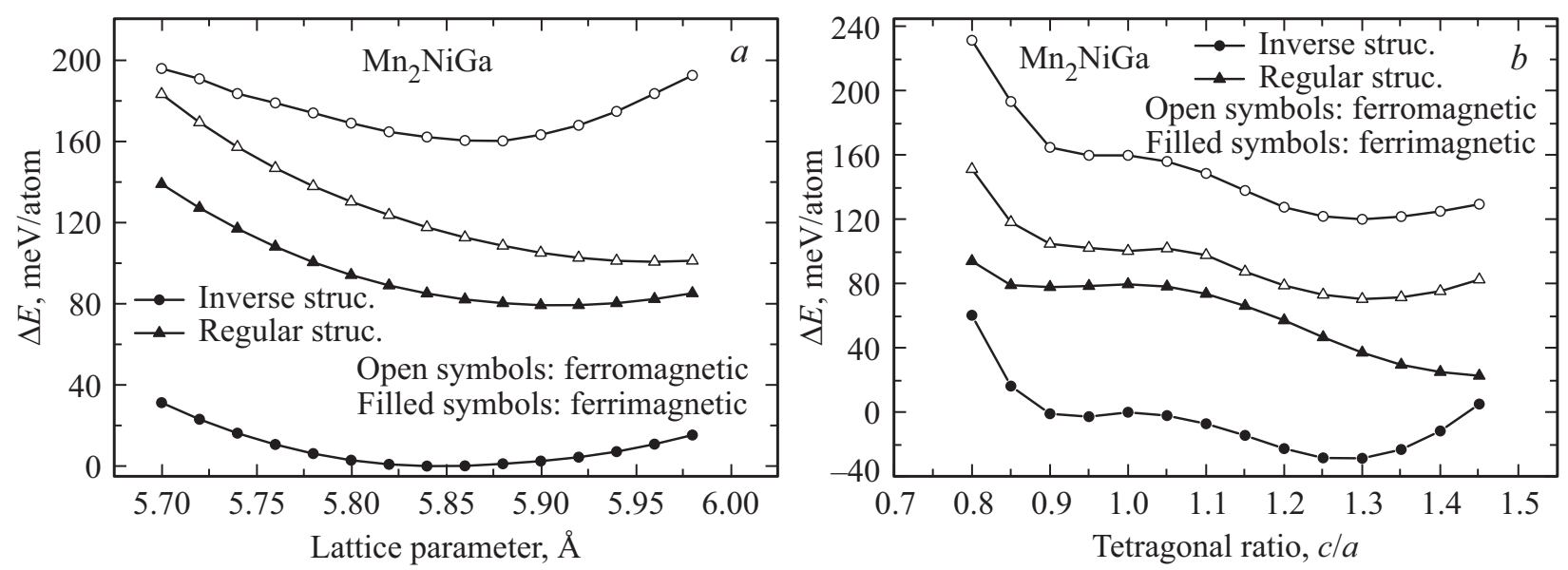

Рис. 4. Изменение полной энергии как функций (a) параметра решетки и $(b)$ степени тетрагональности с/a для Mn2NiGa c учетом ферромагнитного и ферримагнитного спинового упорядочения в ОГС и РГС. Изменение энергии приведено по отношению к ОГС с ферримагнитным упорядочением.

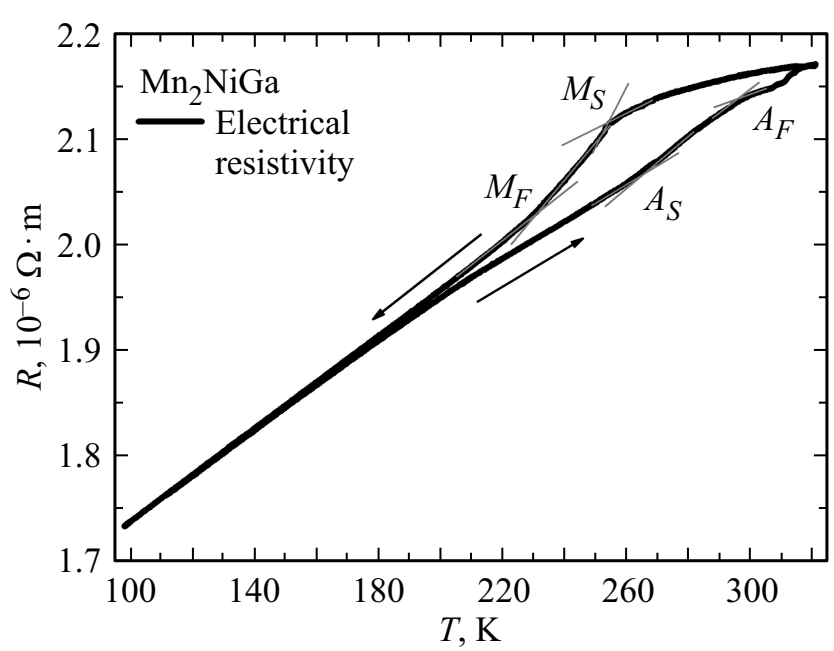

Рис. 5. Зависимость электросопротивления термообработанного образца сплава $\mathrm{Mn}_{2} \mathrm{NiGa}$ от температуры при нагреве и охлаждении в области мартенситного ФП. Методом экстраполяции определены температуры начала и конца прямого и обратного ФП.

момента аустенитной и мартенситной фазы составляют $1.09 \mu_{B} /$ f.u. и $0.95 \mu_{B} /$ f.u. соответственно. Оценка температуры мартенситного перехода $T_{m}$ из разности энергий аустенита $\left(E_{a}\right)$ и мартенсита $\left(E_{m}\right)$ (в meV/atom): $T_{m}=\left(E_{a}-E_{m}\right) / k_{B}$, где $k_{B}-$ постоянная Больцмана, дает $T_{m} \approx 315 \mathrm{~K}$.

Для уменьшения температурного гистерезиса магнитных свойств сплава $\mathrm{Mn}_{2} \mathrm{NiGa}$ применялся дополнительный отжиг. В соответствии с данными DSC (рис. 2) была выбрана температура отжига $1073 \mathrm{~K}$, при которой в течение $72 \mathrm{~h}$ отжигались образцы, с последующим остыванием в печи.

После термообработки исследовалась зависимость электросопротивления образцов от температуры в ши- роком интервале температур $100-320 \mathrm{~K}$, результаты измерений представлены на рис. 5. По этим данным методом экстраполяции определялись температуры начала и конца прямого $M_{S}=254 \mathrm{~K}, M_{F}=231 \mathrm{~K}$ и обратного $A_{S}=266 \mathrm{~K}, A_{F}=296 \mathrm{~K}$ мартенситного ФП. Таким образом, можно утверждать, что термообработка образцов сплава привела к увеличению температур ФП и уменьшению температурного гистерезиса.

Исследования ЭПФ проводились вблизи температур мартенситного ФП сплава при помощи метода трёхточечного изгиба $[11]$ на образце в форме пластинки размерами $0.5 \times 2.0 \times 7.0 \mathrm{~mm}$ при постоянных механических нагрузках от 10 до $247 \mathrm{MPa}$. Характерные зависимости деформации образца сплава $\mathrm{Mn}_{2} \mathrm{NiGa}$ от температуры при термоциклировании представлены на рис. 6.

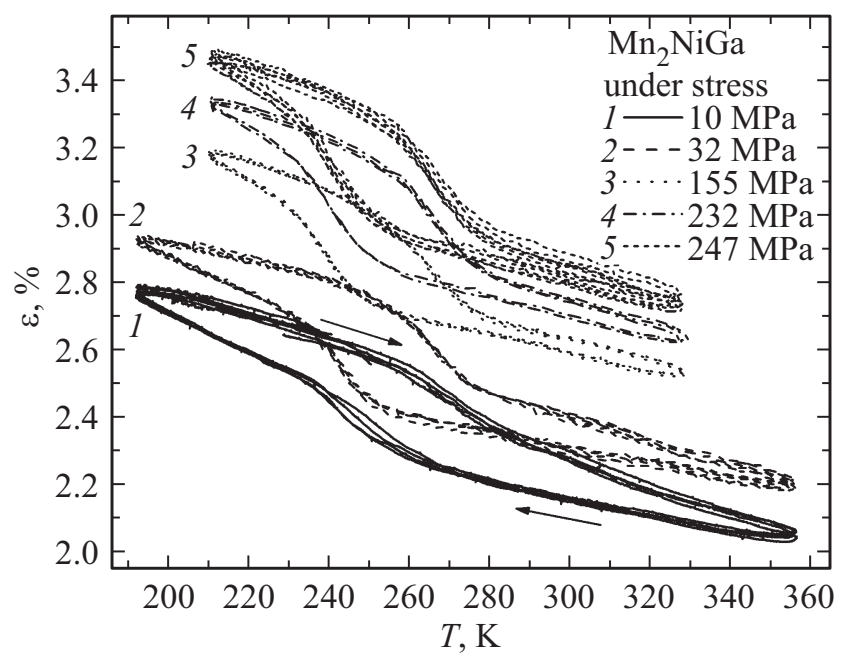

Рис. 6. Зависимость деформации термообработанного образца сплава $\mathrm{Mn}_{2} \mathrm{NiGa}$ от температуры (при 3-х кратном термоциклировании) под действием различных механических нагрузок от 10 до $247 \mathrm{MPa,} \mathrm{полученная} \mathrm{при} \mathrm{помощи} \mathrm{метода}$ трехточечного изгиба [11]. 
Зависимость обратимой деформации от механической нагрузки при обратном термоупругом мартенситном ФП представлена на рис. 7. При увеличении нагрузки не происходит заметного увеличения обратимой деформации, а сама зависимость не является линейной. Тем не менее, сплав демонстрирует значения обратимой деформации до $0.35 \%$.

Зависимость температур ФП в сплаве $\mathrm{Mn}_{2} \mathrm{NiGa}$ от нагрузки представлена на рис. 8. При изгибающих механических нагрузках от 10 до $247 \mathrm{MPa}$ температуры ФП незначительно смещаются в область более высоких температур. Отметим, что при небольших нагрузках до $10 \mathrm{MPa}$ для сплава характерен II тип термоупругого мартенситного ФП [16], когда обратный мартенситный ФП протекает „преждевременно“ ниже $T_{e q}-$ температуры термодинамического равновесия фаз, и темпе-

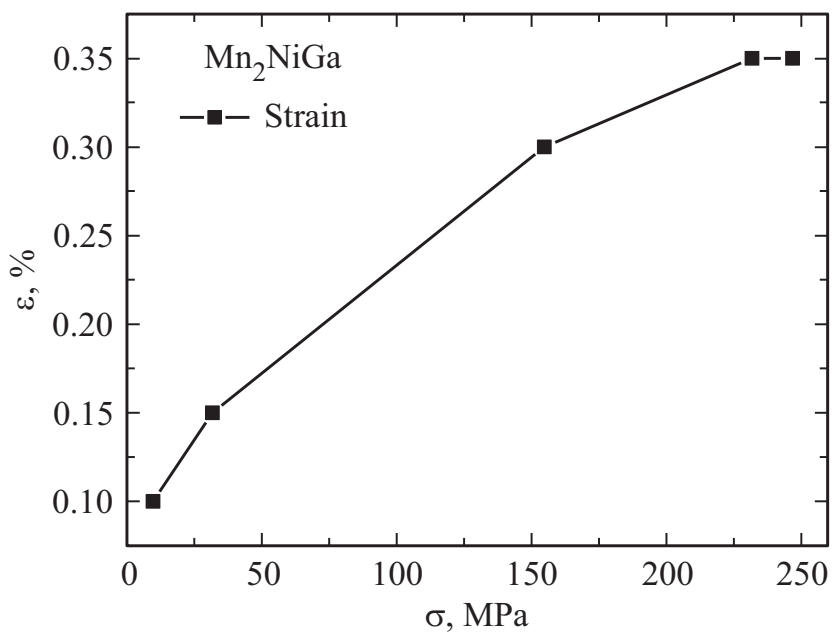

Рис. 7. Зависимость обратимой деформации термообработанного образца сплава $\mathrm{Mn}_{2} \mathrm{NiGa}$ при обратном термоупругом мартенситном ФП от механической нагрузки.

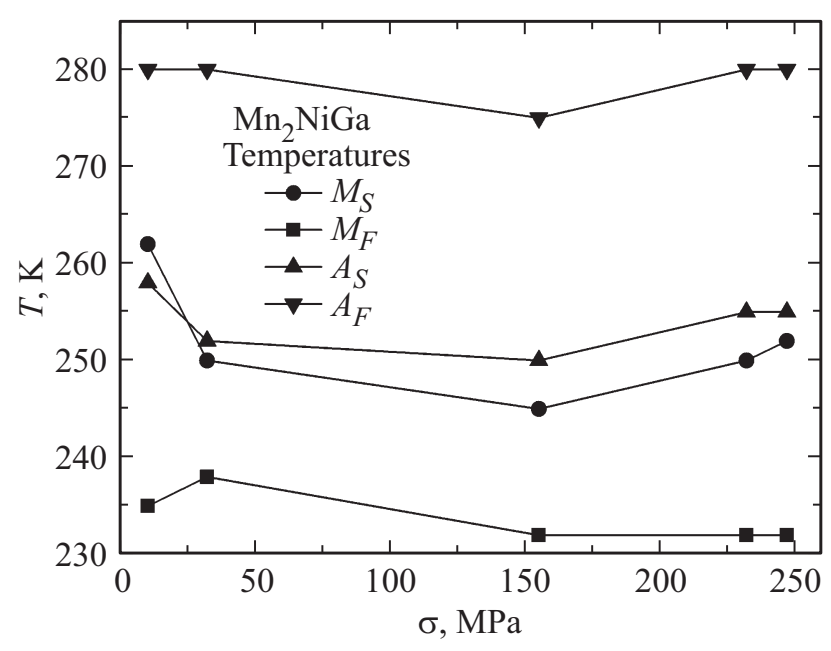

Рис. 8. Зависимость температур прямого $\left(M_{S}, M_{F}\right)$ и обратного $\left(A_{S}, A_{F}\right)$ термоупругого мартенситного ФП в сплаве $\mathrm{Mn}_{2} \mathrm{NiGa}$ от механической нагрузки.

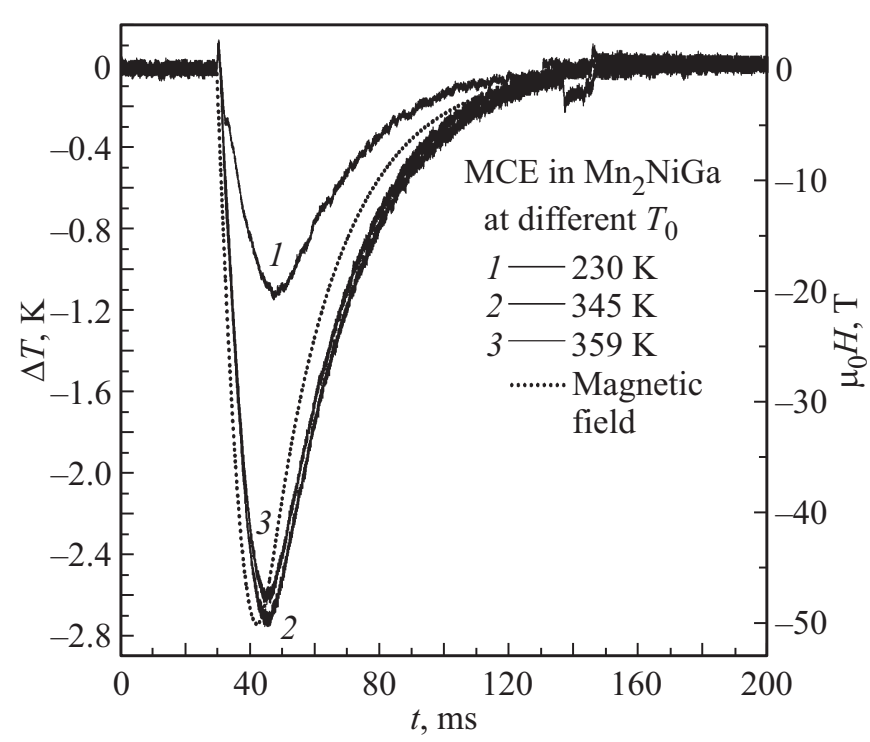

Рис. 9. Временна́я зависимость изменения температуры образца сплава $\mathrm{Mn}_{2} \mathrm{NiGa}$ при разных начальных температурах в магнитном поле до $50 \mathrm{~T}$ (левая шкала), и временно́й профиль магнитного поля - пунктиром (правая шкала).

ратура $A_{S}$ находится ниже температуры $M_{S}$. При увеличении нагрузки происходит смена типа превращения на I. Различия в определении температур ФП методами электросопротивления (рис. 5) и трехточечным изгибом, объясняются влиянием наведенных напряжений в образце при испытаниях на изгиб.

Исследования МКЭ в сильных магнитных полях проводились по следующему протоколу: предварительно образец быстро охлаждался от комнатной температуры до $100 \mathrm{~K}$, а затем нагревался до требуемой (начальной) температуры вблизи ФП. После стабилизации температуры подавался импульс магнитного поля в $50 \mathrm{~T}$. Магнитное поле до $50 \mathrm{~T}$ нарастало за $13 \mathrm{~ms}$, а затем спадало до 0Т в течение примерно $100 \mathrm{~ms}$ (на рис. 9 временной профиль магнитного поля показан сплошным серым цветом на фоне и соответствует правой шкале).

При разных начальных температурах ниже и выше области ФП получены отрицательные значения МКЭ при нарастании поля до $50 \mathrm{~T}$, то есть в сплаве $\mathrm{Mn}_{2} \mathrm{NiGa}$ наблюдается обратный МКЭ, как в мартенситной, так и в аустенитной фазе (рис. 9), что не характерно для сплавов с РГС $[17,18]$, которые могут демонстрировать обратный МКЭ в слабомагнитной мартенситной фазе и прямой МКЭ в ферромагнитной аустенитной фазе. Что также характерно, при начальной температуре ниже $M_{F}$ во внешнем магнитном поле образец сплава с РГС будет охлаждаться до какого-то значения поля $(\approx 6 \mathrm{~T}$ в работе [17]), а при дальнейшем увеличении поля начнётся нагрев образца, а при начальной температуре выше $A_{F}$ сплавы с РГС демонстрируют прямой положительный МКЭ [18].

В сильных импульсных магнитных полях до $50 \mathrm{~T}$ также обычно наблюдается насыщение обратного МКЭ. 
Например, для сплава $\mathrm{Fe}_{49} \mathrm{Rh}_{51}$ с ФП типа АФМ-ФМ максимум обратного МКЭ наблюдается в магнитном поле $\approx 22 \mathrm{~T}$, а при дальнейшем росте поля до $50 \mathrm{~T}$ температура образца возрастает на $1 \mathrm{~K}[19]$.

Таким образом, сплав Гейслера $\mathrm{Mn}_{2} \mathrm{NiGa}$ показывает нетипичное магнитокалорическое поведение - обратный МКЭ как в мартенситной, так и в аустенитной фазах, и при этом МКЭ в сильном импульсном магнитном поле 50 T не испытывает насыщения (рис. 8), что объясняется наличием ферримагнитного упорядочения в сплаве. В то же время, значения обратного МКЭ в сплаве $\mathrm{Mn}_{2} \mathrm{NiGa}$ не велики - максимальное полученное значение составило $\Delta T=-2.7 \mathrm{~K}$ при начальной температуре $345 \mathrm{~K}$ в магнитном поле $50 \mathrm{~T}$ (рис. 8), что значительно ниже по величине, чем у РГС $[17,18]$, а также ниже, чем у других известных магнитокалорических материалов с избытком $\mathrm{Mn}[20,21]$.

\section{4. Заключение}

Было показано, что сплав Гейслера $\mathrm{Mn}_{2} \mathrm{NiGa}$ действительно обладает ферримагнитным упорядочением, а в области мартенситного ФП проявляет гистерезисное поведение в намагниченности и электросопротивлении. Данный сплав также демонстрирует температурный ЭПФ, и при обратном термоупругом мартенситном ФП показывает значения обратимой деформации до $0.35 \%$ при изгибающих механических нагрузках до $247 \mathrm{MPa}$. Кроме того, обнаружен обратный МКЭ как в мартенситной, так и в аустенитной фазах, который не испытывает насыщения, в импульсном магнитном поле до 50 T (т. е., не меняется знак эффекта). Максимальное зафиксированное значение МКЭ для сплава составило $\Delta T=-2.7 \mathrm{~K}$ при начальной температуре $345 \mathrm{~K}$ в поле $50 \mathrm{~T}$.

\section{Благодарности}

Авторы выражают благодарность за поддержку Hochfeld-Magnetlabor Dresden (HLD) Helmholtz-Zentrum Dresden-Rossendorf (HZDR), члену Европейской лаборатории магнитных полей (EMFL)."

\section{Финансирование работы}

Работа выполнена в рамках государственного задания и частично поддержана Российским фондом фундаментальных исследований (проекты № 17-07-01524, 18-08-01434). Теоретические расчеты выполнены В.В. Соколовским за счет Российского научного фонда (проект № 17-72-20022).

\section{Список литературы}

[1] G.D. Liu, J.L. Chen, Z.H. Liu, X.F. Dai, G.H. Wu, B. Zhang, X.X. Zhang. Appl. Phys. Lett. 87, 262504 (2005).

[2] G.D. Liu, X.F. Dai, S.Y. Yu, Z.Y. Zhu, J.L. Chen, G.H. Wu, J.Q. Xiao. Phys. Rev. B 74, 054435 (2006).

[3] T. Graf, S.S.P. Parkin, C. Felser. Prog. Solid State Ch. 39, 1 (2011).

[4] S. Singh, R. Rawat, S.E. Muthu, S.W. D’Souza, E. Suard, A. Senyshyn, S. Banik, P. Rajput, S. Bhardwaj, A.M. Awasthi, R. Ranjan, S. Arumugam, D.L. Schlagel, T.A. Lograsso, A. Chakrabarti, S.R. Barman. Phys. Rev Lett. 109, 246601 (2012).

[5] S. Singh, S.E. Muthu, A. Senyshyn, P. Rajput, E. Suard, S. Arumugam, S.R. Barman. Appl. Phys. Lett. 104, 051905 (2014).

[6] L. Ma, W.H. Wang, J.B. Lu, J.Q. Li, C.M. Zhen, D.L. Hou, G.H. Wu. Appl. Phys. Lett. 99, 182507 (2011).

[7] H.F. Tian, J.B. Lu, L. Ma, H.L. Shi, H.X. Yang, G.H. Wu, J.Q. Li. J. Appl. Phys. 112, 033904 (2012).

[8] L. Ma, S.Q. Wang, Y.Z. Li, C.M. Zhen, D.L. Hou, W.H. Wang, J.L. Chen, G.H. Wu. J. Appl. Phys. 112, 083902 (2012).

[9] H.C. Xuan, S.C. Ma, Q.Q. Cao, D.H. Wang, Y.W. Du. J. Alloy. Compd. 509, 5761 (2011).

[10] Y. Yoshimine, D. Mitsunaga, H. Orihashi, D. Shimada, M. Hiroi, R. Onodera, S. Kimura, K. Takahashi, K. Koyama. IEEE Trans. Magn. 50, 1001204 (2014).

[11] V.S. Kalashnikov, V.V. Koledov, D.S. Kuchin, A.V. Petrov, V.G. Shavrov. Instrum. Exp. Tech. 61, 306 (2018).

[12] M. Ghorbani Zavareh, C. Salazar Mejía, A.K. Nayak, Y. Skourski, J. Wosnitza, C. Felser, M. Nicklas. Appl. Phys. Lett. 106, 071904 (2015).

[13] G. Kresse, J. Furthmüller. Phys. Rev. B 54, 11169 (1996).

[14] G. Kresse, D. Joubert. Phys. Rev. B 59, 1758 (1999).

[15] P. Perdew, K. Burke, M. Ernzerhof. Phys. Rev. Lett. 77, 3865 (1996).

[16] Д. Перкинс, Г.Р. Эдвардс, С.Р. Сач. В сб.: Эффект памяти формы в сплавах. Металлургия, М. (1979). С. 230.

[17] E.T. Dilmieva, Yu.S. Koshkid'ko, A.P. Kamantsev, V.V. Koledov, A.V. Mashirov, V.G. Shavrov, V.V. Khovaylo, M.V. Lyange, J. Cwik, L. Gonzalez-Legarreta, H.B. Grande. IEEE Trans. Magn. 53, 2503705 (2017).

[18] R. Fayzullin, V. Buchelnikov, M. Drobosyuk, A. Mashirov, A. Kamantsev, B. Hernando, M. Zhukov, V. Koledov, V. Shavrov. Solid State Phenomena 233-234, 183 (2015).

[19] A.P. Kamantsev, A.A. Amirov, Yu.S. Koshkid'ko, C. Salazar Mejía, A.V. Mashirov, A.M. Aliev, V.V. Koledov, V.G. Shavrov. Phys. Solid. State 62, 160 (2020).

[20] F. Guillou, H. Yibole, A. Kamantsev, G. Porcari, J. Cwik, V. Koledov, N.H. van Dijk, E. Brück. IEEE Trans. Magn. 51, 2503904 (2015).

[21] Yu.S. Koshkid'ko, E.T. Dilmieva, J. Cwik, K. Rogacki, D. Kowalska, A.P. amantsev, V.V. Koledov, A.V. Mashirov, V.G. Shavrov, V.I. Valkov, A.V. Golovchan, A.P. Sivachenko, S.N. Shevyrtalov, V.V. Rodionova, I.V. Shchetinin, V. Sampath. J. Alloy. Compd. 798, 810 (2019).

Редактор Т.Н. Василевская

\section{Конфликт интересов}

Авторы не имеют конфликта интересов. 